$(1)^{2}+y$

\section{5}

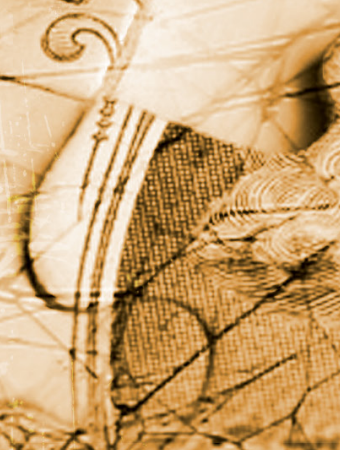

(

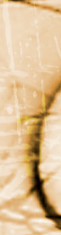

$x \rightarrow x^{2}=1$

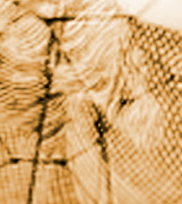

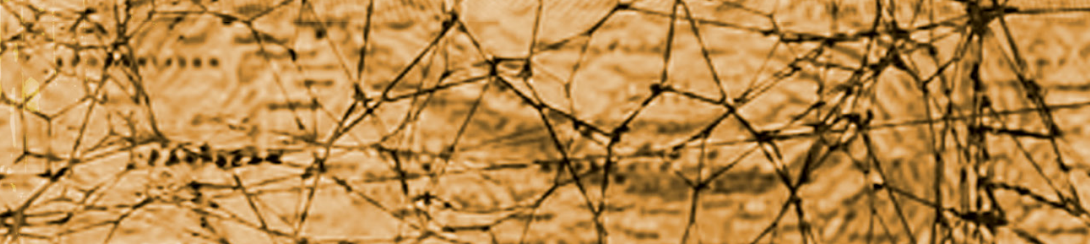

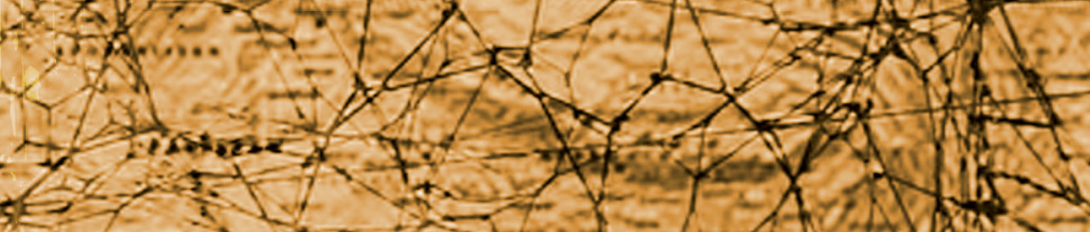

I 301$)^{3}$

WA:GI
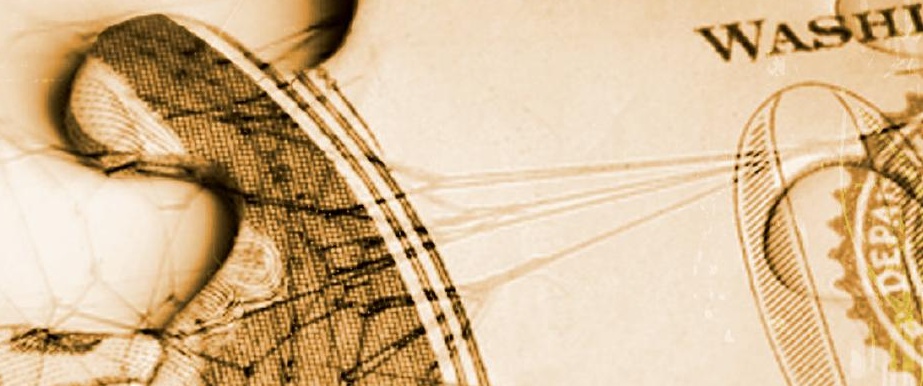

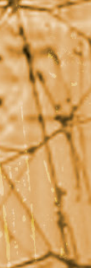

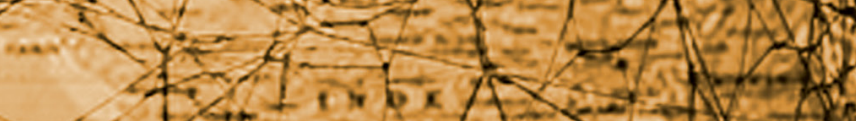

$-1-5$

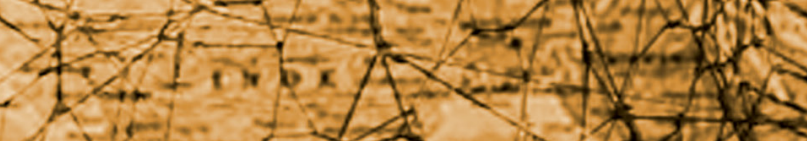

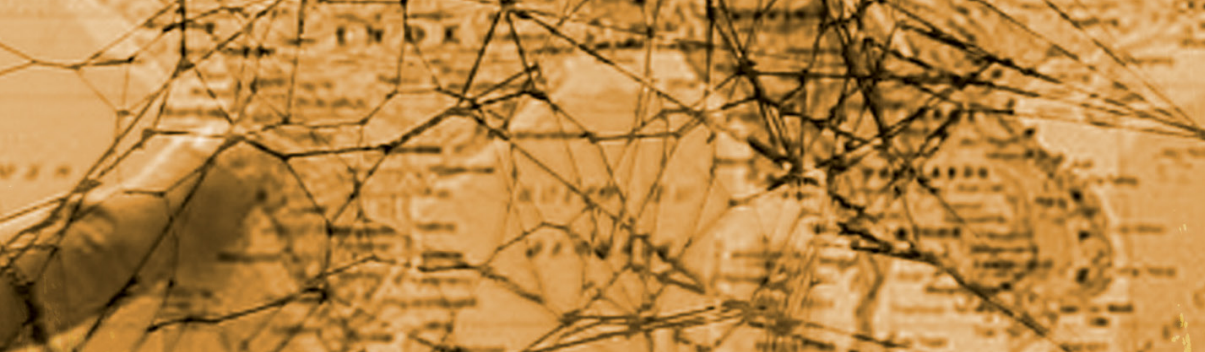

to

(2)

(2) kN

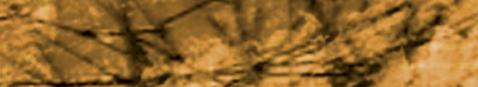




\title{
Comprender las entrañas de las redes invisibles del capi- talismo conectivo y global ${ }^{1}$ FUCHS, Christian (2014). Social Media. A Critical introduction. London: Sage ISBN 978-1-4462-5731-9 (pbk), 978-1-4462- 5730-2 (hbk)
}

DOI: $10.15213 /$ REDES.N12.P398

FELICIANO CASTAÑNO VILLAR

\begin{abstract}
"La experiencia para los bárbaros es algo que tiene la forma de sirga (cuerda), de secuencia, de trayectoria: supone un movimiento que encadena puntos diferentes en el espacio de lo real: es la intensidad de esa chispa... adquirir una experiencia de las cosas se convierte en pasar por ellas justo el tiempo necesario para obtener de ellas un impulso que sea suficiente para acabar en otro lado... el mutante ha aprendido el tiempo mínimo y máximo, que debe demorarse sobre las cosas. $Y$ esto lo mantiene inevitablemente lejos del fondo" Baricco (2008, p. 114-115).
\end{abstract}

Hoy, sin postergar demasiado, tenemos sobre el tapete la necesidad y la urgencia de comprender los medios tecnológicos de la información y comunicación. Requerimos de un conocimiento profundo que aprenda a relacionarse con las contradicciones y los debates del cambiante y complejo mundo de lo ya se denomina hegemónicamente la red. A partir de aquí, podremos hacer análisis fundamentados que nos abran las puertas a entender y participar sobre lo que acontece en el panorama de los medios, los dispositivos y las aplicaciones conectivas. Para aquellas personas que se dejen acoger y azuzar por la pedagogía de la pregunta y la sospecha, de la mano de la filosofía, antropología y sociología neo-marxista de la tecnología, el libro aquí reseñado, así como su autor será un buen aliado.

Christian Fuchs, aún con exceso de didactismo en presentación y fondo, formula convenientemente preguntas a hacernos sobre los medios digitales e Internet, profundizando en el estudio de plataformas como Google, Facebook, Twitter, WikiLeaks y Wikipedia. El resultado es una completa revisión acerca

\footnotetext{
1 - Texto realizado en el marco del proyecto del plan nacional de I+D La ciudadanía en los nuevos escenarios digitales y escolares: relaciones e implicaciones en el alumnado de la educación secundaria obligatoria, referencia EDU2010-18585.
} 
de los medios y su sociología, dejando al descubierto las estructuras y relaciones de poder desiguales, en el triunfante paisaje contemporáneo de las globalizaciones digitales. Es de considerar el esfuerzo de Fuchs por introducir el pensamiento marxista y neo-marxista en los estudios sobre sociología de los medios, incluso como introducción para no iniciados en su primer capítulo. El mundo de las pantallas y las tele-redes suele interpretarse y generar a su alrededor posibilidades y características etéreas que, a modo de repetirse una y otra vez machaconamente por las instancias publicitarias, mediáticas y académicas, suelen afianzar-naturalizar una serie de valores y propiedades ajenos a las prácticas efectivas del día a día, encubriendo con el humo de un discurso del ciberespacio como edén democratizante, liberador y colaborativo los atropellos contemporáneos que sufren nuestras sociedades. La perspectiva materialista, alejada de miradas totalizantes e idealismos crédulos, trata de dibujar los contornos de ese difícil lugar que son hoy las tele-redes sociales. No obstante, sería más rico incluir en la dieta intelectual, en beneficio de una mayor comprensión y transformación de Internet y los medios, otras perspectivas que, aún no siguiendo la senda de la economía política, pudieran arrojar luz sobre un fenómeno cambiante de múltiples caras y tecnicidades.

Christian Fuchs, es uno de esos científicos sociales implicados en la institucionalización de la investigación neo-marxista de Internet y las redes digitales. Desarrolla y promueve revistas de acceso abierto, impulsa conferencias, así como lugares y tiempos para la discusión y construcción de la sociología crítica de la tecnología y los medios. Para más, nada mejor que ver los contenidos de sus plataformas y canales: http://fuchs.uti.at/

En su obra constata la relevancia que hoy tiene el derecho de acceso a la información, al conocimiento público y a la investigación científica. Siendo éstos catalizadores para ejercer la participación social y política y fortalecer la educación de los ciudadanos y de sus profesionales, contribuyendo a la democratización de la sociedad. Al mismo tiempo, garantizar los derechos a la información y al conocimiento supone combatir fenómenos como el cabildeo y el soborno, edificando la pluralidad de las sociedades y la preservación del patrimonio in-material e historiográfico. Por todo ello, Fuchs y una corriente de investigación civil y académica, persigue desvelar las mitificaciones y visiones deterministas y descontextualizadas que se re-producen a la hora de estudiar las redes tecnológicas para, cuanto antes, desde el conocimiento crítico, comenzar a "reinventar Internet, descolonizarla y transformarla en una verdadera esfera pública. Sobre la base de Mahatma Gandhi, que cuando se le preguntó ¿qué piensa de la civilización occidental?, respon- 
dió 'creo que sería una buena idea'. Podemos decir hoy: los medios de comunicación social serían una buena idea si se tratara de público verdaderamente libre. Los medios de comunicación social son posibles, pero por el momento siguen siendo una blochiana que aún no es." Fuchs (2014a).

Internet y los medios tecnológicos de comunicación están inevitablemente interponiéndose en las diferentes actividades humanas, prácticas de razonamiento y disquisición, lectura, interpretación y expresión, organización social y política, ocio, consumo y producción, relación y comunicación interpersonal, experiencias afectivas y sexuales, expresión artística-cultural, etc., afectando a todas las dimensiones de la vida. El ciberespacio da lugar a una vida distinta (una "segunda vida"), siguiendo a Lessig (2009, p. 147). Pero igualmente, los sujetos y la sociedad con sus diferentes acciones, disposiciones y asimétricos campos de fuerza colaboran en la construcción de los media y el ciberespacio. Esta relación dialéctica, compleja y contradictoria entre la tecnología y la sociedad queda patente a lo largo de la exposición teórica y empírica de los diferentes casos analizados por Fuchs, siguiendo la estela de la filosofía crítica de la tecnología de Feenberg (2002, 2011). Para ello, el autor, recoge y actualiza de la teoría crítica de Marx, Engels y Habermas fundamentalmente, con contribuciones de Adorno, Horkheimer, Lukács, Marcuse, Boltanski-Chiapello y Harvey, entre otros, el enfoque marxista de la economía política, los estudios de comunicación política y herramientas conceptuales como ideología, acumulación, valor de cambio, plusvalía, explotación, dominación, desigualdad, poder y esfera pública, incorporando convenientemente pinceladas del feminismo, el igualitarismo del movimiento obrerista o la teoría del capitalismo periférico/central y desvelando a la par los contextos históricos, circunstanciales y las relaciones asimétricas de producción-consumo que se dan en Internet y los media. En la obra se incluyen debates teóricos que desde la sociología, los estudios culturales británicos y comunicacionales se han dado con mayor énfasis (Dean, Murdock, Mosco, Garnham, Gladwell, Morozov, Christensen, etc.). De la misma forma, recoge controversias manifestadas o que incisivamente introduce el autor, con algunos ejemplos, (Castells, Jenkins, Shirky, Papacharissi), mostrando la polarización de las interpretaciones entre ciber-entusiastas (integrados) y ciber-detractores (apocalípticos), que, desde paradigmas idealistas, utilizan explicaciones esencialistas y mecanicistas que poco contribuyen al análisis riguroso de las tecnologías informacionales y conectivas.

La obra argumenta contra la falacia de la democracia participativa basada en la web, profesada por Jenkins, reduciendo la participación de sus signifi- 
cados culturales y escindiéndose asépticamente de la teoría de la democracia participativa. Jenkins obvia el papel central de la propiedad y una democracia participativa es lo contrario a una monopolización de la propiedad y el control. Del mismo modo, y a partir del concepto habermasiano, discute sobre si Twitter es una esfera pública constatando sus límites. Examina los fenómenos de igualdad de voz que exige la comunicación política y la igualdad de acceso a los recursos en la red creadora de tendencias.

Fuchs recoge sintéticamente los debates de Shirk y Papacharissi, por un lado, y el de Morozov, Dean y Gladwell, por otro. Los primeros consideran a los medios sociales como herramientas de una esfera pública en progreso, mientras que los segundos proclaman que el slacktivism (activismo perezoso) desplaza la acción política concreta-encarnada, reafirmando con ello el status quo mientras mitiga los sentimientos de culpa. Fuchs se aproxima al punto de vista escéptico, sin dejar de ver las potencialidades de las capacidades de uso. Para mostrar las acotaciones públicas de la esfera Twitter acude al estudio empírico de la comunicación política y la economía política, recogiendo datos de las revoluciones ciudadanas de la primavera árabe, el movimiento Occupy y el quincemallismo español en Twitter. El autor señala que, si bien las redes sociales pueden ser una herramienta de las movilizaciones sociales, perdura la visibilidad desigual, favoreciendo a las grandes compañías empresariales y a las célebres figuras políticas. Una cuestión nuclear a incluir en el rico examen de la esfera pública que desarrolla Fuchs sobre Twitter sería profundizar en los mecanismos ocultos que utiliza la empresa para seleccionar las tendencias (trending topic).

El autor retoma la definición marxista de explotación, vinculada a una plusvalía basada en la rentabilidad indirecta generada por la conexión, la creación de contenidos y los usos de los llamados prosumers, así como la mano de obra que requiere la economía de la información y el conocimiento. Para Fuchs, a diferencia de Jenkins, la producción de valor del usuario, más allá del tiempo de trabajo, sea buscado o no (produzca satisfacción o no) es explotación. El autor explica con astucia la inexistencia del botón "no me gusta" de Facebook, no tanto por la generación de una cultura positiva y eficaz sino, más bien, por el aumento de valor que supone el no rechazar a nada ni nadie dentro de un espacio informacional y publicitario indiferenciado. En un presente, donde el impacto de la automatización en las personas y la dependencia conectiva asciende (CARR, 2014), de aparente y esterilizado minimalismo mediático, en cuya cúspide se alza Apple, es muy estimable la centralización de la lógica del capitalismo que desarrolla Fuchs frente a las visiones trufadas y funestas. No obstante, intuyo que al autor se le escapa que 
el capitalismo (total) de nueva generación despliega un poder político ilimitado, que maximiza los espacios de competencia produciendo con ello una subjetividad inédita en los sujetos (siguiendo a Laval y Dardot, 2013) y cuerpos. Aunque la plusvalía indirecta generada por los navegantes es irrebatible (por medio de información-publicidad-datos), en un mundo sobre-representado por esferas de competencia expandida, pasar de la plusvalía mediática a la explotación sin más, requiere de muchos matices y tramas no detallados.

Internet y los medios "eléctricos", capaces de transformar los mensajes y contenidos, (como decía McLuhan en 1964 (1996)), están repletos de atribuciones desmesuradas, re-producidas de modo implícito y explícito en multitud de formatos discursivos. De este modo se van implantando acríticamente en el imaginario colectivo toda una serie de falsas características propias de Internet y sus diferentes plataformas como Google, Facebook o Twitter. Clichés y espejismos, como libertad, democracia, colaboración, cultura participativa, innovación y esfera pública, van acumulándose en los sentidos comunes. La aceptación de teorías deterministas, el cortoplacismo y el sobrevalorado don de la rapidez no permiten trascender las inmediatas aspiraciones del beneficio, equivocando con facilidad la notoriedad con el ser o la potencialidad con la verdadera apropiación y capacidad de uso y repercusión de los medios de tele-comunicación social.

$\mathrm{El}$ autor deja patente que conceptualizaciones como sociedad de redes o sociedad de la información de Castells, nos hacen creer que hemos entrado en un nuevo tipo de sociedad, invisibilizando, desde su teoría mayúscula, las problemáticas de exclusión, desigualdad y dominación que suponen un modelo espacial, temporal, transnacional vinculado a un régimen flexible de acumulación a cambio de mostrar las redes digitales como algo inmóvil. Éstas conceptualizaciones no logran mostrar la negatividad y el carácter de clase de estos cambios, descuidando así nociones fundamentales como dominación, explotación, poder e ideología (Fuchs 2008).

Fuchs prefiere hablar de capitalismo informacional transnacional o global, con el objeto de subrayar la dialéctica de continuidad y discontinuidad y el rol de la información y los nuevos medios tecnológicos de comunicación en la sociedad. En el modo informacional del capitalismo, la plusvalía y la acumulación de capital se manifiestan cada vez más en mercancías "inmateriales" y en trabajo cognitivo, comunicativo, cooperativo y afectivo (siguiendo en esto a Hardt y Negri). La acumulación de capital, poder y capacidades a escala mundial están intensamente arbitradas por las tele-redes. 
"Internet y los medios de comunicación son el espejo de las estructuras de explotación y opresión de energía que encontramos en la sociedad contemporánea." (FUCHS, 2014b, p. 202-204). Es por ello que la célebre brecha digital no es digital, sino el reflejo de la estructura de clases sociales (etnias, sexos-géneros, capacidades) del capitalismo informacional transnacional. Para ello el autor aporta múltiples datos que atestiguan desequilibrios geográficos y sociales en el uso de las redes digitales. Las redes tecnológicas transforman los medios de producción y sus relaciones, el conocimiento se produce, comparte y consume con la ayuda de los media. En esta dinámica dialéctica, el conocimiento y la tecnología se reafirman mutuamente.

Fuchs muestra acertadamente como los derechos de privacidad actuales favorecen a la privacidad de la élite financiera y política, mientras que perjudican desproporcionadamente a la intimidad individual. Quizá por ello Edward Snowden se haya convertido en el malhechor más buscado en el mundo. En el libro, WikiLeaks es concebido como instrumento de transparencia cuya expresión es de naturaleza social y procomún, mientras el almacenamiento de datos de clientes de Facebook, distribuido de manera poco clara para los anunciantes, es entendido como vigilancia. Wikileaks es identificado como herramienta sobrecargada de ideología liberal, al centrar su injerencia sobre las fuerzas del gobierno (principalmente sobre la corrupción) frente a la inmanente explotación del sistema capitalista y sus corporaciones así como a las mediaciones existentes entre las transnacionales y los gobiernos.

Wikipedia, a diferencia de las compañías digitales y de telecomunicaciones, tienen una naturaleza verdaderamente socialista y comunitaria. Es de código abierto, basado en los comunes (CC, Creative Commons) y la construcción comunitaria (edición colaborativa), su publicidad es gratuita y está disponible sin valor de cambio alguno salvo la satisfacción del uso.

Fuchs no vacila en concluir que las verdaderas tecnologías comunitarias-sociales, aquellas que estén al servicio de las necesidades y los deseos de la humanidad, serán sólo posibles en una sociedad comunista, una sociedad de los comunes. Añadir a su reflexión, el reto que propone Lanier (2011), cambiar de paradigma para dar preferencia a la subjetividad humana frente a la tecnología como último bien de salvación. 


\section{REFERENCIAS BIBLIOGRÁFICAS}

BARICCO, A. (2008). Los bárbaros. Ensayo sobre la mutación. Barcelona: Anagrama.

CARR, N. (2014). The Glass Cage, Automation and Us. New York: Norton Company.

FEENBERG, A. (2002). Transforming Technology: A Critical Theory Revisited. Oxford University Press.

FUCHS, C. (2008). Internet and society: Social theory in the information age. Nueva York: Routledge.

FUCHS, C. (2014a). (Un-) Freedom in the Age of Social Media. En http://fuchs. uti.at/1023/ (Última consulta: 29 de mayo de 2014)

FUCHS, C. (2014b). Social Media. A Critical introduction. London: Sage.

LANIER, J. (2011). Contra el rebaño digital. Barcelona: Debate

LAVAL, C. Y DARDOT, P (2013). La nueva razón del mundo. Barcelona: Gedisa. LESSIG, L. (2009). El código 2.0. Madrid: Traficantes de sueños.

MCLUHAN, M. (1996). Comprender los medios de comunicación. Las extensiones del ser humano. Barcelona: Paidós Comunicación. 